\title{
Advanced Adaptivity in Learning Management Systems by Considering Learning Styles*
}

\author{
Sabine Graf and Kinshuk \\ School of Computing and Information Systems, Athabasca University, Canada \\ sabineg@athabascau.ca; kinshuk@athabascau.ca
}

\begin{abstract}
Typical learning management systems consider only little or, in most cases, no adaptivity. In this paper, we introduce an adaptive mechanism which enables such systems to provide students with courses that fit their individual learning styles. The adaptive mechanism is based on an advanced student modelling approach which identifies learning styles by automatic, dynamic, and global student modelling. Based on the identified learning styles, the adaptive mechanism composes courses that match the students' learning styles, aiming at making learning easier for students. Furthermore, the adaptive mechanism aims at being easy to use for teachers by being generic and adaptable for teachers, allowing them to adjust the mechanism to their course structure and preferences.
\end{abstract}

\section{Introduction}

Nowadays, more and more educational institutions, such as universities, offer e-learning courses. Such elearning courses need an environment, where they are managed and organised. In the majority of cases this task is fulfilled by a learning management system (LMS). LMSs aim at supporting teachers in creating, administering, and managing online courses by providing them with a variety of features. However, LMSs typically do not consider individual differences of learners and treat all learners equally regardless of their needs and characteristics.

But the individual learners play a central role in traditional as well as technology enhanced learning. Each learner has individual needs and characteristics such as different prior knowledge, cognitive abilities, learning styles, motivation, and so on. These individual differences affect the learning process and are the reason why some learners find it easy to learn in a

\footnotetext{
* The authors acknowledge the support of Austrian Science Fund (FWF) under grant J2831-N13, iCORE, Xerox, and the research related gift funding by Mr. A. Markin.
}

particular course, whereas others find the same course difficult [1].

In this paper, we focus on incorporating learning styles in LMSs. Research about considering learning styles in technology enhanced learning is motivated by educational and psychological theories, which argue that learners have different ways in which they prefer to learn. Furthermore, Felder, for example, pointed out that learners with a strong preference for a specific learning style may have difficulties in learning if the teaching style does not match with their learning style [2, 3]. From theoretical point of view, conclusion can be drawn that incorporating students' learning styles in the learning environment makes learning easier for them and increases their learning efficiency. On the other hand, learners whose learning styles are not supported by the learning environment may experience problems in the learning process.

This paper introduces a mechanism for enabling LMSs to provide advanced adaptivity for learners through incorporating their learning styles according to the Felder-Silverman learning style model (FSLSM) [2]. At the same time, the mechanism keeps the use of the system easy for teachers and allows them to adapt the mechanism to their preferences rather than forcing them to modify their courses to the requirements of the adaptive mechanism.

FSLSM is one of the most often used learning style models in technology enhanced learning. It combines major learning style models such as the model by Kolb [4], Pask [5], and the Myers-Briggs Type Indicator [6]. FSLSM uses the concept of dimensions and therefore describes learning styles in much detail. Each learner has a preference on each of the four dimensions: active/reflective, sensing/intuitive, visual/verbal, and sequential/global. Learning styles, as described by FSLSM, are considered as "flexibly stable" [7], which means that they are more or less stable but can change over time, for example, if a learner trains his/her weak learning preferences. Furthermore, FSLSM is based on the concept of tendencies which means that even a 
learner with, e.g., a strong active learning style can act sometimes in a reflective way.

The adaptive mechanism is developed in a general way but implemented as part of a larger project [8], which aims at exploring and developing different applications and content delivery systems for building a personalised mobile learning environment. Other applications and systems include a location awareness grouping service, context-awareness service, multimedia input service, question \& answer service, problem-based learning service, and social network service. These applications and services are combined with the LMS Moodle [9], for which the adaptive mechanism is implemented.

The adaptive mechanism is based on an advanced student modelling approach in order to identify the students' learning styles. The different applications and systems from the learning environment provide rich information about students' behaviour and actions which is then used as input for the student modelling approach, explained in more detail in the next section. Subsequently, the generic framework of the adaptive mechanism is explained. In Section 4, the provision of adaptive courses is discussed in more detail and Section 5 concludes the paper.

\section{Identifying learning styles}

In order to provide adaptivity, the characteristics of learners have to be known first. This paper proposes an advanced student modelling approach which identifies learning styles according to the FSLSM. Investigations are based on previous research work [10], where an automatic and static student modelling approach has been designed, implemented, and evaluated. The evaluation showed that the approach is suitable for identifying learning styles and therefore can be seen as the basis for the development of an advanced student modelling approach which combines automatic, static, dynamic, and global student modelling.

Automatic student modelling means that the process of building and updating the student model is done automatically based on the behaviour and actions of the learners when they are using the system for learning. Therefore, students need to focus only on learning rather than additionally providing explicit feedback about their preferences. While the students are learning, the system tracks their behaviour and actions and uses this information for inferring learning styles by applying a rule-based method. The automatic part of the student modelling approach is based on previous work [10] but is extended by additionally incorporating patterns from the different services and applications in the learning environment. Therefore, more information about students' behaviour and actions can be gathered and included in the student modelling approach, which leads to higher accuracy due to more available data.

The dynamic part of the student modelling approach is responsible for using the information about students' behaviour and actions for updating the student model frequently while students are using the system for learning. Therefore, the student modelling approach is able to improve and revise the information about students' learning styles once contradictory data from students' behaviour and actions are found, leading to a higher accuracy of the identified learning styles and subsequently, more accurate adaptivity.

The global aspect of student modelling focuses on considering all components and services integrated in the learning environment for gathering data about the students' behaviour and actions. Therefore, more data about students' behaviour and actions are available, allowing more accurate identification of learning styles.

In addition, we offer students the possibility to fill out the Index of Learning Styles questionnaire [3], a well-known instrument for identifying learning styles according to the FSLSM. If a student filled out this questionnaire, the student model can be initialised by the data from the questionnaire, while data about the student's behaviour and actions can then be used for revising and improving the information from the questionnaire, allowing the approach to learn the student's learning styles and identify changes in his/her learning style preferences.

Figure 1 depicts the architecture of the student modelling approach. The student modelling approach uses input data from the applications and services of the learning environment as well as from the learning style questionnaire, if data are available. Based on the input data, it calculates and updates the students' learning styles and stores the results in the student

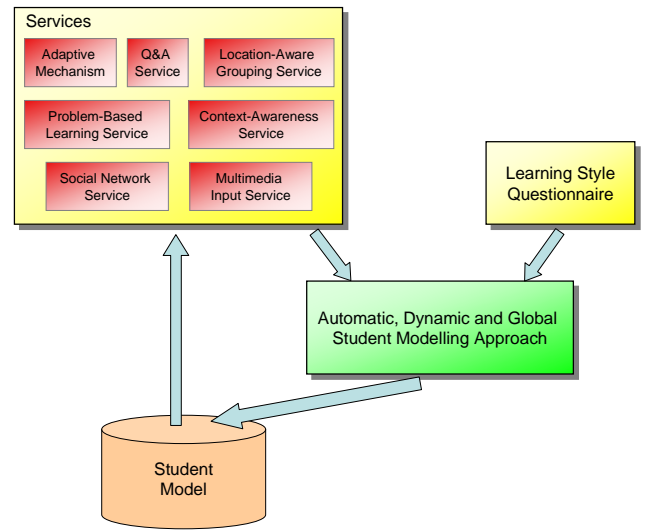

Figure 1. Architecture for student modelling 
model. The calculated learning styles can be accessed by all applications and services in order to provide learners with adaptivity.

\section{A generic framework of the adaptive mechanism}

Once learning styles are known, learning systems can be extended in a way that they are able to automatically generate and present courses that fit the students' learning styles. For providing adaptive courses, most adaptive systems ask for a specific course structure and specific types of learning objects in order to apply their adaptation rules and generate adaptive courses. In this paper, we present an adaptive mechanism which is generic and therefore allows teachers to suit the adaptive mechanism to their available course in terms of course structure and used types of learning objects. Instead of requiring a predefined course structure, the generic adaptive mechanism is adaptable by teachers and allows them to incorporate any types of learning objects, ranging from simple types such as outlines, examples, and exercises to any component and service which can be used for providing learners with learning experiences.

In order to facilitate teachers to specify the incorporated types of learning objects, teachers are supported by a configuration tool that enables them to describe, on the one hand, the types of learning objects which should be considered in the adaptive mechanism as well as how the adaptive mechanism should use the types of learning objects in order to provide different courses for students with different learning styles.

When creating a new type of learning object in the adaptive mechanism, the tool allows teachers to specify the following data: First, information about which learning styles can be supported by the new type has to be provided (e.g., a digital reading room supports a reflective learning style). Second, teachers have to be able to state how this type of learning object is represented in the learning system (e.g., a digital reading room is represented in the learning system by the component digital reading room and an example is represented in the learning system by the component resource). Third, suitable adaptation features need to be specified for the new type in the adaptive mechanism. Adaptation features indicate how a course can change for students with different learning styles. The tool provides a set of predefined strategies in order to facilitate teachers in specifying adaptation features. For example, predefined strategies include provide $X$ before $Y$ and provide a high number of $X$, where $\mathrm{X}$ and $\mathrm{Y}$ are types of learning objects in the adaptive mechanism. Additionally, the tool asks teachers to specify for which learning style the new adaptation feature has to be applied.

Once a new type of learning object is specified in the configuration tool of the adaptive mechanism, the authoring tool, which is used for the creation of learning objects, is automatically extended in order to provide teachers with the possibility to specify that a created learning object is of this new type. Furthermore, the expert model, where the learning objects are stored, is extended respectively in order to store the new type of learning object. Based on these extensions, the adaptive mechanism can distinguish between and know the types of all available learning objects, as they are specified in the configuration tool. Additionally, the authoring tool and expert model is extended in a way that they allow specifying a type of learning object which can be excluded from adaptivity and displayed at the same place for all students.

\section{Provision of adaptive courses}

The proposed adaptive mechanism uses the gathered information from the student modelling approach to provide adaptive courses. Figure 2 presents the architecture of the adaptive mechanism integrated in an LMS. The adaptation module is the core element of the adaptive mechanism. It is connected to the student module, expert module, interface module, and configuration tool and uses information from these modules/tools to compose courses that fit the students' individual learning styles. Similar to intelligent tutoring systems, the expert module stores the available learning objects and information about their types, the student module provides information about the students' learning styles, and the interface module is responsible for interacting with students. Furthermore, the configuration tool provides information about the incorporated types of learning objects and the adaptation features which indicate how different types of learning objects should be used for learners with different learning styles.

In previous research work [11], a concept for composing adaptive courses based on predefined types of learning objects and predefined adaptation features was introduced. However, the proposed adaptive mechanism in this paper is more advanced, using a generic framework for providing adaptivity rather than restricting teachers to a specific course structure and integrating an automatic, dynamic, and global student modelling approach rather than using simply a learning style questionnaire for identifying learning styles. 


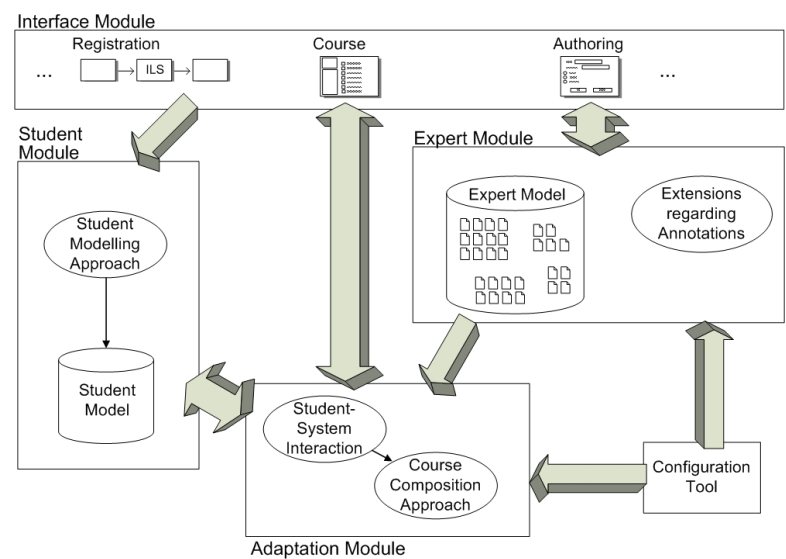

Figure 2. Architecture of the adaptive mechanism integrated in an LMS

In order to incorporate the dynamic aspect of the student modelling approach, the system is able to interact with the students. When using a dynamic student modelling approach, information in the student model is modified, revised, or improved frequently. Based on the concept of providing adaptivity, a modification in the student model implies a change of the student's course. In order to avoid confusing students, the system interacts with the students once a change in their learning styles is noticed, informing them about the identified change. Then the system recommends a new adaptive course and asks the student whether he/she accepts the modification and is comfortable with it.

\section{Conclusions and Future Work}

In this paper, we presented an adaptive mechanism which enables LMSs to provide advanced adaptivity with respect to learning styles. The adaptive mechanism is based on an automatic, dynamic, and global student modelling approach, gathering data about students' behaviour and actions from different components and services and calculating and frequently updating the students' learning styles based on this information. A main objective of the adaptive mechanism is to be generic and adaptable to the teachers' needs. Therefore, teachers are supported in the task of specifying which learning objects should be included in their courses. Based on this information, the adaptive mechanism provides learners with adaptive courses and interacts with students once it recognises changes in their learning styles.

The proposed adaptive mechanism aims at making learning easier for students by providing them with courses that fit their individual learning styles. Furthermore, the mechanism aims at being easy to use for teachers, letting them continue using their already available courses in LMSs by allowing adjustments in the mechanism to the characteristics of the respective courses in order to provide them as adaptive ones.

Future work will deal with completing the implementation of the adaptive mechanism and evaluating it. The effectiveness and the usability of the adaptive mechanism will be evaluated within the larger project and data from different applications and services will be used for evaluating the student modelling approach.

\section{References}

[1] D. H. Jonassen and B. L. Grabowski, Handbook of Individual Differences, Learning, and Instruction, Lawrence Erlbaum, Hillsdale, New Jersey, 1993.

[2] R. M. Felder and L. K. Silverman, Learning and teaching styles in engineering education, Engineering Education, 78, 1988, pp. 674-681.

[3] R. M. Felder and B. A. Soloman, 1997. Index of Learning Styles questionnaire. Retrieved 25 April, 2009, from http://www.engr.ncsu.edu/learningstyles/ ilsweb.html

[4] D. A. Kolb, Experiential learning: Experience as the source of learning and development, Prentice-Hall, Englewood Cliffs, New Jersey, 1984.

[5] G. Pask, "Styles and strategies of learning," British Journal of Educational Psychology, vol. 46, 1976, pp. $128-148$

[6] I. Briggs Myers, Manual: The Myers-Briggs Type Indicator. Consulting Psychologists Press, Palo Alto, CA, 1962.

[7] F. Coffield, D. Moseley, E. Hall, and K. Ecclestone, Learning Styles and Pedagogy in Post-16 Learning: A Systematic and Critical Review. Learning and Skills Research Centre/University of Newcastle upon Tyne, London, 2004.

[8] S. Graf, K. MacCallum, T.-C. Liu, M. Chang, D. Wen, Q. Tan, J. Dron, F. Lin, R. McGreal, and Kinshuk, An Infrastructure for Developing Pervasive Learning Environments, Proceedings of the IEEE International Workshop on Pervasive Learning (PerEL 2008), Hong Kong, March 2008, pp. 389-394.

[9] Moodle, 2009. Retrieved 15 April, 2009, from http://www.moodle.org

[10] S. Graf, Kinshuk, and T.-C. Liu, Identifying Learning Styles in Learning Management Systems by Using Indications from Students' Behaviour, Proceedings of the International Conference on Advanced Learning Technologies, IEEE Computer Science, Los Alamitos, 2008, pp. 482-486.

[11] S. Graf and Kinshuk, Providing Adaptive Courses in Learning Management Systems with Respect to Learning Styles, Proceedings of the World Conference on E-Learning in Corporate, Government, Healthcare, and Higher Education, AACE Press, 2007, pp. 25762583. 